Heinz-Dieter Pohl ${ }^{*}$

Universität Klagenfurt

UDK 811.163.6:811.112.2(497.452+436.5)

DOI: 10.4312/linguistica.60.2.179-191

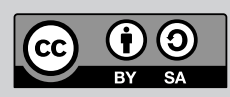

\title{
SLOWENISCH-DEUTSCHER SPRACHKONTAKT, GEZEIGT AN DER SPRACHINSEL ZARZ/SORICA IM VERGLEICH MIT KÄRNTEN
}

\section{ALLGEMEINES}

\section{1}

Während der deutsche Einfluss aufs Slowenische bestens dokumentiert ist (z. B. Kranzmayer 1944 und Striedter-Temps 1963), findet man über den slowenischen Einfluss aufs Deutsche Angaben vornehmlich in Spezialarbeiten zu deutschen Mundarten (z. B. Lessiak 1944), wenn man von Arbeiten aus der Zeit der Österreichisch-Ungarischen Monarchie absieht (z. B. Lessiak 1910/1983, Štrekelj 1909). Die slowenischen Einflüsse auf die Deutschkärntner Mundart sowie auf die Volks- und Umgangssprache haben Neweklowsky (1990) und ich wiederholt dargestellt (eine Zusammenfassung meiner Arbeiten dazu ist in Pohl 2009 erschienen).

Deutsch als überregionale Verkehrssprache in der österreichischen Reichshälfte der Österreichisch-Ungarischen Monarchie und Slowenisch als bodenständige Volksund Umgangssprache Krains, der Südsteiermark und von großen Teilen Kärntens koexistierten jahrhundertelang und haben einander auf verschiedene Weise beeinflusst (vgl. Krevs Birk 2019). Während der dt. Einfluss im Slowenischen allgemein ist, also fast das gesamte Sprachgebiet sowohl auf Ebene der Volkssprache als auch der älteren Schriftsprache betrifft, beschränkte sich der sl. Einfluss aufs Deutsche auf die Volkssprache im Umkreis des sl. Sprachgebietes und nur bis zu einem gewissen Grad auch auf die Umgangssprache (heute v. a. noch in Kärnten, dazu vgl. Pohl 1993, 1997 u. 2009), ist also regional. Besonders intensiv war der sl. Einfluss auf die dt. Sprachinselmundarten im Tal der Selzacher Zaier (sl. Seľ̌čica), in Zarz (sl. Sorica), Deutschrut (sl. Nemški Rut) und Huben (sl. Spodnje Danje). Bezüglich des benachbarten Zaierfeldes (sl. Sorško polje zwischen Krainburg/Kranj und Bischoflack/Škofja Loka) hat dies schon Valvasor für Feichting (sl. Zg./Sp./Sr. Bitnje) festgestellt (zitiert nach Lessiak 1944: 25f.):

Unter denselben / höret man dreyerley Sprachen; als die rechte creinerische / rechte Teutsche / und / drittens / die aus der Crainerisch und Teutschen unter einander gemengte.

Jedoch wird die / also gemischte / nur allein in dem Dorff Feichting geredt: welches insgemein Bitina genannt wird... Wer die Leute dieses Dorffs verstehn soll /

heinz.pohl@chello.at 
der muß beydes wol Crainerisch / und wol Teutsch / können: weil die Einwohner dieses langen Dorffs im Reden / beydes ineinander mengen.

Diese „Feichtinger Mischsprache“ soll sich in einigen Familien bis Anfang des vorigen Jahrhunderts gehalten haben (Lessiak 1944: 25 Anm. 3). Eine vergleichbare Sprachform hat Kranzmayer noch 1941 in Huben (sl. Spodnje Danje) aufgenommen (veröffentlicht in Lessiak 1944: 219); die Textproben werden hier in Kap. 2 (mit Erläuterungen) geboten.

\section{2}

Zum sl. Einfluss auf die dt. Sprachinselmundarten in Krain vgl. v. a. Lessiak (1944: passim, Wortschatz 75ff., WB 17ff.). In den von Lessiak (1944: 202ff.) zusammengestellten Sprachproben der Krainer dt. Sprachinselmundarten fand ich folgende bemerkenswerte Interferenzen:

\subsubsection{Satzkonstruktion ohne es}

brt furt nåuxgäibm ce žnāeban (WS 2) 'es wird sofort nachgeben (= aufhören) zu schneien'; šaent šwåxe cāetn/šsent šwåxe cāetn/šaent šläxte cāetn (WS 13) 'es sind schlechte (schwache) Zeiten'; haent nåxt hout gežnibm (WS 25) 'heute Nacht hat es geschneit'. Solches ist im Kärntner Deutsch gang und gäbe, z. B. rēgnet 'es regnet', hait wår khålt 'heute war es kalt' (vgl. Pohl 2009: 126 bzw. Pohl 1993: 655 mit Lit. u. weiteren Beispielen).

\subsubsection{Zeitformen}

Das Perfekt (als allgemeines Präteritum) wird nur mit sein gebildet, z. B. de ōltn lāete žint genoumen ... 'die alten Leute haben genommen' (sind nach sl. Vorbild, das im Präteritum nur sein als Verbum auxiliare kennt, ${ }^{1}$ vgl. 2.8., 2.10 u. 2.14); desgleichen das Futurum, z. B. ..., lež de milix drbōalala 'damit die Milch zu sieden beginnt' (wörtlich 'aufwallen wird', s. 2.1). Das Hilfsverbum sl. bom, boš usw. 'werde (sein)' fehlt dabei oft (wie u.a. in 2.1). Es wird beim Verbum sein im Dt. dann durch werden allein wiedergegeben, z. B. ...wer i pāme hāože 'werde ich beim Hause (sein oder bleiben)' (nach sl. Vorbild bom, boš usw. 'ich werde sein'). Beides ist auch in Kärnten zu beobachten, z. B. $i($ ch) pin ferschlå:fn 'ich habe mich verschlafen', ...wer $i$ pis åchte då: 'ich werde bis $8^{\text {h }}$ da (sein)' (vgl. Neweklowsky 1990: 490 u. 491). Das typisch Kärntnerische ich bin geschlafen (statt habe) dürfte aber damit nicht zusammenhängen, denn schlafen bedeutete ursprünglich 'schlaff liegen', und liegen hat im Perfekt sein (und nicht wie im nördlichen Deutschen haben).

1 Die Bildung des Präteritums und Futurums erfolgt im Sl. mit dem gemeinslaw. l-Partizip, sspr. $-l[\mathrm{w}]$ (m.), -la (f.), -lo (n.), pl. -li, -le, -la. Die ins Sl. entlehnten dt. Verba werden wie die sl. Verba auf -ati flektiert, wobei das m. Partitizip -al zu ma. [ow] wird, die anderen Formen regulär: -ala, -alo, -ali usw. 


\subsubsection{Lehnbeziehungen und -bedeutungen}

Vielfach wird dt. aber (ma. åfr) im Sinne von 'und' (wie im Sl. pa) verwendet. Das Adjektiv dt. schwach hat (analog zu sl. slab) auch die Bedeutung 'schlecht' angenommen (vgl. WS 13). Sl. Lehnwörter sind nicht selten, darunter allgemein-südbairische wābe, Kärnten Wābm 'Frau, Weib (pejorativ)' oder auf Krain beschränkte wie kesle 'Stückchen', Diminutiv zu sl. kós 'Stück, Teil' (mit dt. Umlaut < *kösle).

\subsubsection{Historisches (s. a. 1.1)}

Die Besiedlung des Gebiets durch (süd-)bairisch sprechende Bauern aus dem Hochpustertal um Innichen (Tirol) erfolgte um 1200, mehr als hundert Jahre vor der Besiedlung der Gottschee/Kočevje. Bis ins frühe 20. Jahrhundert wurde in Zarz/Sorica ein altertümlicher südbairischer Dialekt gesprochen. Trotz der deutschen Amtssprache im alten Österreich ging der Gebrauch des Deutschen in Zarz angesichts der immer enger werdenden Beziehungen mit der slowenischsprachigen Umgebung schon im Laufe des 19. Jahrhunderts zurück. Die Volkszählung 1910 (publ. 1918), noch in Österreich-Ungarn, wies Zarz/Sorica bereits als überwiegend slowenischsprachig aus. In Huben/Spodnje Danje bestand noch längere Zeit eine Mischsprache mit deutschem Vokabular und slowenischer Grammatik (einige Bemerkungen zur Geschichte dieser „Hubner Mischsprache“ s. bei Lessiak 1944: 67, 25ff.). Da das Slowenische 1945 bereits von allen Bewohnern von Zarz/Sorica gesprochen wurde, waren diese nicht von der Vertreibung der deutschen Minderheit im Rahmen der AVNOJ-Beschlüsse betroffen. Bergnamen deutscher Herkunft wie Altemaver $(<$ ålte māur 'Alte Mauer') oder Tonderškofel ( $<$ tondrškhouvl 'Donnerskofel') und Gewässernamen wie Driselpoh (< drišlpåx 'Drischelbach') oder Štajnpoh (< štōampåx 'Steinbach', auch Siedlungsname für Podrošt, eigentlich 'Untere Rast' zu dt. ma. råšt 'Rast(platz)') sind bis heute in Gebrauch, ebenso Haus- und v. a. Familiennamen.

\subsubsection{Umschrift}

Die von mir verwendete Umschrift für die Krainer dt. Sprachinselmundarten, v. a. in Kap. 2, ist gegenüber dem Original (s. Lessiak 1944: 218f.) vereinfacht und für dt. wie sl. Wörter gleich (ohne Berücksichtigung der Unterschiede zwischen den 3 Klassen von Spiranten, vgl. Lessiak 1944: 81, was hier irrelevant ist; $s$ stimmloses, $z$ stimmhaftes $s, c=t s, w=$ bilabial (ähnlich wie engl. $w$ ), $x=c h, \bar{e} \bar{q}$ langes offenes $e o$; die übrigen Zeichen dürften keine nähere Erläuterung benötigen). Im Dt. wird sl. $b$ oft als $w$, umgekehrt dt. $w$ im Sl. als $b$ wiedergegeben. Da das dt. ma. bilabiale $w$ fast wie stimmhaftes $b$ gesprochen wird, ist es in den Textproben auch so geschrieben. Der historische und z. T. hochsprachliche $b$-Laut wurde (v. a. im An- und Auslaut) zu $p$. 


\section{TEXTPROBEN (,HUBNER MISCHSPRACHE“ NACH LESSIAK 1944: 219 BZW. 7)}

2.1 (WS 3) dāj khol in oufm, lež de milix drbōalala.

dāj (sl.) 'gib' (Imperativ 2.Psg.); khol (dt.) 'Kohle' (ma. n.); in oufm (dt.) 'in den Ofen'; lež (dt.) 'dass, damit' (zu als, vgl. WB 34); de milix (dt.) 'die Milch' (wie auch südbair.); drbōolala (dt./sl.) 'sieden' (d. i. dt. ma. drwåln 'erwallen' = 'zu sieden beginnen' + sl. $\left.-a l a^{2}\right)$.

Ü: Gib Kohle in den Ofen, damit die Milch zu sieden (erwallen) beginnt. HD: gip kheldr èn oufn, lež dē milix brt drbåln.

2.2 (WS 5) je fōr fìr pa žekš boxn štęerbow (oder geštōrbm).

je (sl.) 'ist'; fōr (dt.) 'vor', fïr (dt.) 'vier'; pa (sl.) 'aber, und' (hier: 'oder'); žekš (dt.) 'sechs'; boxn (dt.) ‘Wochen' (pl.); štęrrbow (dt./sl.) 'gestorben' (d. i. dt. sterben + sl. $-o w^{3}$ ), alternativ statt dessen das dt. PPP 'gestorben'.

Ü: Er ist vor oder sechs Wochen gestorben.

HD: ęr išt fōr fìrn afr žęešn båxn gestōrbm.

2.3 (WS 6) ce štō̄rkhe fājr je biw, gebęanclar sa (oder žajn) čist šwōrc.

ce (dt.) 'zu'; štōrkhe (dt.) 'stark'; je biw (sl.) 'ist gewesen'; gebęanclar (sl./dt.) ${ }^{4}$ die (Weihnachts-, Oster-)Kuchen'; sa (sl. ma.) 'sind', alternativ statt dessen auch dt. ma. žajn 'sind' (bair. sain(t)); čist (sl.) 'rein' (hier: 'ganz'); šwōrc (dt.) 'schwarz'.

Ü: Das Feuer war zu stark, die Kuchen sind ganz schwarz.

HD: sfāer iškežn (<išt gežen 'ist gewesen') ce štōrkh, de gebęenclar žēnt gotšs šwōrtš.

2.4 (WS 7) iste ōjr ålewajle ounan žōalc pa ounan påpr.

iste (dt.) 'isst + die'; ̄̄jr (dt.) 'Eier'; ålewajle (dt.) 'alleweile; fortwährend, jederzeit' (hier 'immer, stets', bair., vgl. WB 34); ounan (dt.) 'ohne einen' (ohne + unbestimmter Artikel im Sinne des bair. Teilungsartikels); žāolc (dt.) 'Salz' (in Huben/Spodnje Danje m.!, vgl. WB 132); pa (sl.) 'und'; påpr (sl.) 'Pfeffer' (sspr. pōper).

Ü: Er isst die Eier immer ohne (ein) Salz und ohne (einen) Pfeffer.

HD: ęrr išt dōajr štējtšs ('stets') Q̄unan žålc èn ōunan pfêfr.

2 S. o. 1.2.2 u. Anm. 1.

3 S. o. 1.2.2 u. Anm. 1.

4 gebęanclar ist pl. von gewancle 'Weihnachts-, Osterkuchen', Diminutiv zu gewånce, entlehnt aus sl. gubánica 'ds.' (WB 72). 
2.5 (WS 8) fiəse me bę tuənaja, mislim, ka me rīwow.

fiase (dt.) 'Füße'; me (sl.) 'mir'; $b \bar{e}$ (dt.) 'weh' (ma. wēe); tuənaja (dt./sl.) '(sie) tun' (d. i. dt. ma. tuәn + sl. -aja $a^{6}$; mislim (sl.) '(ich) denke, meine'; $k a$ (sl.) 'dass' (eigentlich Relativpronomen); rīwow ${ }^{7}$ (sl.) 'gerieben' (zu sl. ma. ribati, entlehnt aus dt. reiben, vgl. WB 128).

Ü: (Die) Füße tun mir weh, ich meine, dass ich mir (die Haut) aufgerieben habe. HD: de fìse tōant $m r$ bęa, ì mōan, že žent gerīwaet.

\section{6 (WS 13) sa žleaxte cajte}

sa (sl. ma.) '(sie) sind'; žleaxte (dt.) 'schlechte'; cajte (sl.) 'Zeiten' (sl. cajt dt. Lehnwort).

Ü: (Es) sind schlechte Zeiten.

HD: šènt šwåxe csāetn.

2.7 (WS 16) te se net grōs genukh, lež du wån trinkhala a flåša bājna. måraš še båkšow da bla gean grōs.

te (sl.) 'du' (sspr. ti); še (sl.) 'du bist' (sspr. si); net (dt.) 'nicht'; grōs (dt.) 'groß'; genukh (dt.) 'genug'; lež (dt.) 'dass' (s. 2.1); du (dt.) 'du'; wån (sl.) '(hin)aus' (sspr. vèn); trinkhala (dt./sl.), d. i. dt. trinken + sl. -ala ${ }^{8}$; a flåša (dt.) 'eine Flasche'; bājna (dt./ sl.) 'des Weines' (d. i. dt. Wein + sl. Genitiv sg. -a); måraš (sl.) '(du) musst' (sspr. moraš); še (sl.) 'noch'; båkšow (dt./sl.), d. i. dt. ma. båǩ̌n 'wachsen' + sl. -ow'; da (sl.) 'dass, damit', bla (sl.), d. i. sspr. bila, l-Part. zu biti 'sein' (hier Futur ${ }^{10}$ ); geən (dt.) 'gehen' (im Sinne von 'werden', vgl. Lessiak 1944: 187).

Ü: Du bist nicht groß genug, dass du austrinkst eine Flasche Wein. Du musst noch wachsen, damit du größer wirst.

HD: du pišt nox et ('nicht') krōas kēnōakh, lež du āustrīnkhašt a flåše bāan, du måšt nox båkšn èn grēasar geråutn ('geraten').

5 Eigentlich Akk. wie sl. noge me bolijo 'die Füße schmerzen mich'.

6 Ins Sl. entlehnte bzw. slowenisch flektierte dt. Verba werden wie die sl. Verba auf -ati flektiert (s. o. 1.2.2 u. Anm. 1). Hier liegt der Infinitiv tun der sl. Flexion zu Grunde; in dieser Verbalklasse lautet die sl. ma. 3. Ppl. -aja, 3. Psg. - $a$.

7 S. o. 1.2.2 u. Anm. 1.

8 S. o. 1.2 .2 u. Anm. 1.

9 S. o. 1.2 .2 u. Anm. 1.

10 S. o. 1.2 .2 u. Anm. 1. 
2.8 (WS 19) kadù je me štäilow flājš?

kadù (sl.) 'wer' (sspr. kdo, ma. kedo); je (sl.) 'ist' (hier: 'hat'); ${ }^{11}$ me (sl.) 'mir' (sspr. mi); štäilow (dt./sl.) 'gestohlen' (d. i. dt. stehlen + sl. -ow ${ }^{12}$ ); flājš (dt.) 'Fleisch'.

Ü: Wer hat mir das Fleisch gestohlen?

HD: bęr et ('hat') mar s flāeš keštouln?

2.9 (WS 23) me sma miade pa sma durštikh.

me sma (sl.) 'wir sind' (sspr. mi smo); miade (dt.) 'müde'; pa (sl.) 'und'; durštikh (dt.) 'durstig'.

Ü: Wir sind müde und sind durstig.

HD: bìar žêmmīade (< žên mìade) $n \check{z} \bar{a} \bar{e} n$ durštikh.

2.10 (WS 25) žnajbala je hājte nōxt, dam ęršst žnęb je plajbow, hajt šmōranš je dräiprow (oder dręaprow).

žnajbala (dt./sl.) 'geschneit' (d. i. dt. ma. schnaibm + sl. -ala ${ }^{13}$ ); je 'ist', ${ }^{14}$ hājte (dt.) 'heute'; nōxt (dt.) 'Nacht'; dam ęrršt (dt.) 'zuerst' (vgl. WB 58); žnę̣b (dt.) 'Schnee', plajbow (dt./sl.) 'geblieben' (d. i. dt. bleiben + sl. -ow ${ }^{15}$ ); šmōranš (dt.) 'des Morgens' (vgl. WB 112); dräiprow (dt./sl.) 'zerronnen (vom Schnee), aper geworden' (zu dt. ma. dräiprn < (d)er-äpern 'aper (schneefrei) werden' + sl. -ow, ${ }^{1}$ vgl. WB 57).

Ü: Heute Nacht hat es geschneit. Zuerst ist der Schnee liegen geblieben; heute morgen ist er geschmolzen (geapert).

HD: gežnībn ets hāetē nåxt, dām ęarst dr žnēap ǐškeplībm (< išt geplībm), hāet žmōrānš iškęiprt (<išt gęiprt).

2.11 (WS 29) inžəre (oder nāše) ekn sa net ažo hōx kåkr dājre, wāše sa hẹhar (oder bol hōx).

inžare (dt.) 'unsere'; nāšse (sl.) 'unsere'; ekn (dt.) 'Berge' (dt. ma. ekke, vgl. WB 55 $5^{16}$ ); sa (sl. ma.) 'sind'; net (dt.) 'nicht'; ažo (dt.) 'so als (wie)' (also, vgl. WB 35); hōx (dt.) 'hoch'; kåkr (sl.) 'wie' (sspr. kakor); dājre (dt.) 'eure' (eigentlich 'die eueren'), wāše (sl.) 'eure', hęhar (dt.) 'höher', bol hōx (sl./dt.) 'höher' (sl. bolj 'mehr' + dt. hoch).

Ü: Unsere Berge sind nicht so hoch wie die eueren, euere sind höher.

HD: īnžr de pẹrrge žênt et ažou hōaxe, dāere žēnt hēaxar.

11 S. o. 1.2.2.

12 S. o. 1.2.2 u. Anm. 1.

13 S. o. 1.2 .2 u. Anm. 1.

14 S. o. Anm. 6.

15 S. o. 1.2.2 u. Anm. 1.

$16 \mathrm{Vgl}$. Egg in vielen Ortsnamen und -eck in zahlreichen Bergnamen. 
2.12 (WS 33) cwå nāje hājžr drrixta moj prōo(a)dr tam hintr nāšme gōrte.

cwå (dt.) 'zwei'; nāje (dt.) 'neue'; hājžr (dt.) 'Häuser'; drrixta (dt./sl.) '(er) errichtet' (d. i. dt. ma. derrichten, vgl. WB 58, + sl. $-a^{17}$ ); moj (sl.) 'mein', prō(a) $d r$ (dt.) 'Bruder'; tam (sl.) 'dort'; hintr (dt.) 'hinter'; nā̄šme (sl.) 'unserem' (Dativ sg. oder Instrumental sg., sspr. našemu oder našim); gōrte (dt.) 'Garten’.

Ü: Zwei neue Häuser errichtet mein Bruder dort hinter unserem Garten.

HD: māēn dr prōadr māort ('mauert') cwō nāea hāežr drt hīntr inžrme gōrte.

2.13 (WS 34) tu me je wån (oder wan) azme harce paršl.

$t u$ (sl.) 'das' (sspr. to); me (sl.) 'ihm' (sspr. mu), je (sl.) 'ist'; wån, wan (sl.) '(hin) aus' (sspr. vèn); azme (dt.) 'aus dem'; harce (dt.) 'Herzen'; paršl (sl.) 'gekommen' (sspr. prišlo).

Ü: Das ist ihm aus dem Herzen gekommen.

HD: dås išt $m$ ažme harcn khęimên.

2.14 (Lessiak 1944: 7) teste stāre lājte sa nęəmale širbala fājra.

teste (sl.) 'diese' (sspr. tisti); stāre (sl.) 'alten'; läjte (dt.) 'Leute'; sa (sl. ma.) 'sind' (hier: 'haben'18); nęamale (dt./sl.) 'genommen' (d. i. dt. nehmen + sl. -ale ${ }^{19}$ ); šìrbala (dt. ma.) 'Schäufelchen'; fäjra (dt./sl.) '(des) Feuers' (d. i. dt. Feuer + sl. Genitiv sg. - $a$, ähnlich flåša bājna, s. o. 2.7).

Ü: Diese alten Leute haben ein Schäufelchen Feuer genommen.

$\mathrm{HD}:{ }^{20}$ dī̌r (a) åltn lāite nt genoumen a šìrwele fäir.

\section{KOMMENTAR (ZU 2)}

\section{1}

Mit einer einzigen Ausnahme (2.4) sind alle Sätze, grammatikalisch gesehen, mit slowenischem Prädikat gebildet:

2.1 dāj 'gib' und drbōolala 'beginnt zu sieden';

2.2 je ... šterbow (geštōrbm) 'ist gestorben';

2.3 je biw 'ist gewesen', sa (žajn)...šwōrc 'sind schwarz';

2.5 fiase...bę tuanaja 'die Füße tun weh';

$2.6 \mathrm{sa}$ 'es sind';

17 S. o. 1.2 .2 u. Anm. 1.

18 S. o. 1.2 .2 u. Anm. 1.

19 S. o. 1.2 .2 u. Anm. 1.

20 Nach Lexer, ,rein deutsche Mundart“. 
2.7 te se 'du bist', du wån trinkhala 'du wirst austrinken', måraš...båkšow 'du musst wachsen' (auffallend, dass hier eine maskuline Form steht neben zweimaliger femininer), da bla geən grōs 'damit du größer wirst';

2.8 kadù je...štäilow 'wer hat gestohlen';

2.9 me sma 'wir sind';

2.10 žnajbala je 'es hat geschneit', žnęb je plajbow 'der Schnee ist geblieben', je dräiprow 'ist zerronnen';

2.11 inžəre (nāše) ekn sa ... hōx unsere Berge sind hoch', wāše sa hẹher (bol hōx) 'eure sind höher';

2.12 drrixta moj prō(a)dr 'es errichtet mein Bruder';

2.13 je wån...paršl 'ist herausgekommen’;

2.14 läjte sa nęamale ‘die Leute haben genommen'.

Nur 2.4 weist ein deutsches Prädikat auf: iste ōjr '(er) isst die Eier'. Warum dies so ist, kann heute nicht mehr festgestellt werden. Also abgesehen von 2.4 haben wir durchwegs slowenisch konstruierte Sätze vor uns, die reich an deutschem Sprachmaterial sind, doch auch dieses weist vielfach slowenische Merkmale auf, z. B. cajte (2.6), bājna (2.7), bol hōx (2.11), fäjra (2.14), alles deutsche Wörter mit slowenischer Flexion. Dazu kommen slowenische Konjunktionen ( $p a$ besonders oft, ferner $k a, d a$ ). Die Wortfolge entspricht z. T. den deutschen, z. T. den slowenischen Parallelen, geht aber durchaus auch eigene Wege (z. B. 2.12).

\section{2}

Ähnliche Konstruktionen findet man auch in Kärntner Assimilationsgebieten (also solchen Gebieten, in denen das Slowenische dem Deutschen nach und nach weicht), z. B. aus Griffen/Grebinj: jas sm knåp par kāsa 'ich bin knapp bei Kasse' (sspr. jaz sem 'ich bin', dt. knapp, sl. pri, dt. Kassa), oder motor ja häslaufaw 'der Motor ist heißgelaufen' (Beispiele nach Andrej 1980:31). Das letztere entspricht in der Konstruktion genau 2.2/8/10 und mutatis mutandis allen in 3.1 genannten Beispielen. Während die von Andrej (1980: 31) genannten Sätze noch als slowenisch (hinsichtlich der grammatischen Struktur) zu bezeichnen sind, sind Sätze wie 2.2/8/10 als schon slowenisch zu betrachten. Offensichtlich geht der Sprachwechsel nördlich und südlich der Karawanken spiegelverkehrt vor sich: zuerst setzt sich im Norden der (dt.) Wortschatz und dann die (dt.) Grammatik durch, im Süden zuerst die (sl.) Grammatik und später erst der (sl.) Wortschatz. Wahrscheinlich hängt dies mit der Prädominanz der deutschen Sprache und deren höherem Prestige (in Kärnten bis heute, in Krain zumindest bis 1918) zusammen.

\section{3}

Als Rückseite des Spiegels der „Hubner Mischsprache“ finden sich in Kärnten sowohl slowenische Sätze mit deutscher Syntax als auch deutscher Phreaseologie, z. B. qadər snîx praw węlqa sem prída (wörtlich) 'wenn Schnee recht viel daher kommt' (SturmSchnabl 1973: 181) mit konfuser Wortfolge und Phraseologie, oder ân stáwə 'es steht nicht an', puwərxə hòr 'obendrauf' (ebda. 182), beide ad-hoc-Calques, pa da maw qèj 
wən je 'dass etwas draus ist' (gemeint ist '...wird'), qədər pa maw câjta je 'wenn aber etwas Zeit ist' (ebda. 180), mit der dem Dt. nachgebildeten Endstellung von je 'ist', jès hréam lî̀č 'ich gehe liegen' (wie in der dt. Mundart, Isačenko 1939: 132). Ein besonders gutes Beispiel ist an je na tó farbōltar šòw, da sa patrîp tarštélala (ebda., wörtlich) 'und es ist auf das der Verwalter gegangen, dass sie den Betrieb einstellten' (= 'und es ist der Verwalter darauf eingegangen, dass man den Betrieb eingestellt hat'). Deutsches Sprachdenken in slowenischer Verkleidung finden wir u. a. in Sätzen wie wč́:raj brəšt wən bè:w 'gestern ist die Frist aus gewesen' (Karničar 1990: 64).

\section{3}

Slowenische Lehnwörter im Kärntner Deutsch ${ }^{21}$

Jause (< sl. južina 'Mittagessen'; mala južina 'Zwischenmahlzeit' ergab in der sl. Mundart [máwžna] 'Jause'). - Keusche, mundartlich Kaischn 'kleines Bauernhaus, Kate' (< sl. hiša, germanisches Lehnwort). - Poganze $\dagger$ 'ein gefüllter Kuchen (mit Topfen oder Nüssen)' aus Strudel- oder Germteig), ältere Variante von sl. pogača < rom. focatia / focacea, früher eine Art Weißbrot. - Pogatsche $(<$ sl. pogača 'Kuchen, Weißbrot, Festtagsbrot usw.', Herkunft wie Poganzen, in Kärnten vorwiegend für den 'Rein(d)ling' gebraucht; Diminutiv dazu Pogatscherl früher in Wien, heute noch relikthaft Grammelpogatscherl als Weingebäck). - Potitze 'Rollkuchen, Art Reinling' (ein Kuchen aus Germteig mit verschiedenen Füllen, < sl. po(vi)tica 'Rollkuchen'). - Munggen und Talggen † 'einfache bäuerliche Speise aus geschrotetem Getreide' (diese früh entlehnt aus slawisch *tălkъna, russisch toloknó 'Haferbrei, -grütze', polnisch tłokno 'Speise aus Hafermehl, heißem Wasser und Milch'), Munggen (< frühsl. *mo(n)ka 'Mehl', heute sl. moka). - Wābm (ma., umgangssprachlich) 'altes Weib (meist abfällig)' (< sl. baba 'alte Frau'). - Daber 'Klamm, Schlucht' (Osttirol, < sl. deber 'Talschlucht', regional ma. daber). - Huditsch 'Teufel' ( $<$ sl. hudic $)$, als Fluch. - Jaukh † 'Föhn' $(<$ sl. jug 'Süden'). - Koper 'Dille' (< sl. koper). - Maischl, Maischele, -erl 'Netzlaibchen' (rückentlehnt aus sl. majželj < bair. *Maiselein 'kleine Schnitte'). - moidúsch 'meiner Seel' $(<$ sl. (pri) moji duši ). - Patsche / Påtsche 'Eber' $(<$ sl. pačej aus dem Deutschen, zu Bock). - Sāsaka 'Verhacktes, ausgelassener geräucherter Speck' (< sl. zaseka). Schwachta / Schwåchta 'Sippschaft (abwertend)' (< sl. ma. žłahta 'Geschlecht' aus dem Deutschen). - Strankele 'Fisole, grüne Bohne' (< altsl. stro(n)k- 'Schote, Hülse', heute sl. strok). - zwìln (ma., umgangssprachlich) 'klagen, jammern' ( $<$ sl. cviliti).

Bemerkenswert sind die semantischen Gleichungen nach rom. Vorbildern wie Unterdåch 'Dachboden' (wörtlich 'Unterdach' wie sl. podstrešje und furlan. sotèt $<$ rom. subtum tectum) oder Auswart † 'Frühling' (wörtlich 'auswärts', vgl. sl. vigred [wörtlich 'Ausgang'] und furlan. insude $<$ rom. *in-exitus).

21 Näheres Pohl (2009: 127ff.). 


\section{SCHLUSS}

Diese Beispiele zeigen, dass das Erlöschen des Deutschen in Krain und des Slowenischen in Kärnten sehr ähnlich erfolgt. Auf Grund der dt. und sl. Sprachstruktur ergeben sich durchaus gleichwertige Übergangsstadien, die man oft als „Mischsprachen“ bezeichnet hat - in Kärnten als „Windisch“ - ein Wort, das auch andere (v. a. politische) Nebenbedeutungen hat (s. Pohl 2004). Doch in der Tat handelt es sich um Sprachformen, die an Einzelpersonen gebunden sind, die sich im Status assimilationis befinden und im Grunde genommen ,zweisprachig“ im wahr(st)en Sinn des Wortes sind, nicht aber um Gruppensprachen oder gar „Dialekte“ im engeren Sinn des Wortes.

\section{Literatur}

ANDREJ, Johann (1980) Untersuchungen zur Zweisprachigkeit in Griffen und Umgebung. Graz: Universität (unveröffentlichte Hausarbeit).

ISAČENKO, Alexander V. (1939) Narečje vasi Sele na Rožu. Ljubljana: Znanstveno društvo.

KARNIČAR, Ludwig (1990) Der Obir-Dialekt in Kärnten. Die Mundart von Ebriach/ Obirsko. Wien: Österreichische Akademie der Wissenschaften.

KRANZMAYER, Eberhard (1944) Die deutschen Lehnwörter in der slowenischen Volkssprache. Laibach (Ljubljana): Kramarič.

KRANZMAYER, Eberhard/Primus, LESSIAK (1983) Wörterbuch der deutschen Sprachinselmundart von Zarz/Sorica und Deutschrut/Rut in Jugoslawien. Hrsg. v. M. Hornung/A. Ogris. Klagenfurt: Verlag des Geschichtsvereins für Kärnten.

KREVS BIRK, Uršula (2019), Zu einigen Aspekten des Deutschen als Kontaktsprache des Slowenischen." Linguistica 59, 155-173.

LESSIAK, Primus (1910/1983) „Alpendeutsche und Alpenslawen in ihren sprachlichen Beziehungen." Germanisch-Romanische Monatsschrift, 2/1910: 274-288 (nachgedruckt in: Wiesinger, P. [Hg.], Die Wiener Dialektologische Schule. Wien 1983).

LESSIAK, Primus (1944) Die deutsche Mundart von Zarz in Oberkrain. A. Grammatik (mit Ergänzungen von Eberhard Kranzmayer u. Annemarie Richter). Weimar: Böhlau.

NEWEKLOWSKY, Gerhard (1990) „Kärntner Deutsch aus slawistischer Sicht: zum deutsch-slowenischen Sprachbund in Kärnten.“ Germanistische Linguistik 101103: 477-500.

POHL, Heinz-Dieter (1993) „Deutsch-Slowenische Sprachkontakte in Kärnten.“ Carinthia I 183: 651-664.

POHL, Heinz-Dieter (1995) „Slowenisch-deutscher Sprachkontakt in Krain. Bemerkungen zur 'Hubner Mischsprache'.“ In: K. Sornig (et al.) (Hrsg.), Festschrift für Norman Denison zum 70. Geburtstag. Graz: Institut für Sprachwissenschaft der Universität Graz, 315-322.

POHL, Heinz-Dieter (1997) „Österreich.“ In: H. Goebl (Hrsg.), Internationales Handbuch zur Kontaktlinguistik Bd. 2. Berlin: de Gruyter, 1797-1812. 
POHL, Heinz-Dieter (2004) „Sprache und Politik, gezeigt am Glottonym Windisch.“ In: Th. Krisch/ Th. Lindner/U. Müller (Hrsg.) (2005) Analecta homini universali dicata. Festschrift Oswald Panagl zum 65. Geburtstag. Stuttgart: Akademischer Verlag.

POHL, Heinz-Dieter (2005) „Sprachen und Sprachinseln im südalpinen Raum.“ Europa ethnica 2005/3-4: 91-100. http://members.chello.at/heinz.pohl/Sprachinseln. htm.

POHL, Heinz-Dieter (2009) „Sprachkontakt in Kärnten.“ In: M. Elmentaler (Hrsg.) Deutsch und seine Nachbarn. Frankfurt a. Main: Peter Lang, 117-132. http://members.chello.at/heinz.pohl/Sprachkontakt_K.htm.

ŠTREKELJ, Karel (1909) „Slovanski elementi v besednem zakladu štajerskih Nemcev.“ Časopis za zgodovino in narodopisje 5, 38-103, 6, 1-69 u. 112-115.

STRIEDTER-TEMPS, Hildegard (1963) Deutsche Lehnwörter im Slovenischen. Berlin-Wiesbaden: Harrassowitz.

STURM-SCHNABL, Stanislava K. (1973) Die slovenischen Mundarten und Mundartreste im Klagenfurter Becken. Wien: Universität (Dissertation).

\author{
Einige Abkürzungen \\ dt., Dt. deutsch, Deutsch \\ HD Hubner Deutsch \\ ma. mundartlich \\ Ppl. Person Plural \\ PPP Partizip Präteritum Passiv \\ Psg. Person Singular \\ rom. romanisch \\ sl., Sl. slowenisch, Slowenisch \\ sspr. schriftsprachlich \\ Ü Übersetzung \\ WB = Kranzmayer/Lessiak 1983 (s. Literatur) \\ WS Wenker-Satz (Deutscher Sprachatlas zu Marburg)
}

\title{
Zusammenfassung SLOWENISCH-DEUTSCHER SPRACHKONTAKT GEZEIGT AN DER SPRACHINSEL ZARZ/SORICA IM VERGLEICH MIT KÄRNTEN
}

Der slowenisch-deutsche Sprachkontakt in Krain und der deutsch-slowenische Sprachkontakt in Kärnten sind einander recht ähnlich. In beiden Ländern ist es dann vielfach zum Sprachwechsel gekommen, so z. B. in Zarz schon vor 1941/45. Der Sprachwechsel nördlich und südlich der Karawanken ging spiegelverkehrt vor sich: Zuerst setzte sich im Norden der deutsche Wortschatz und dann die deutsche Grammatik durch, im Süden zuerst die slowenische Grammatik und später erst der slowenische Wortschatz. 
Die angeführten Beispiele zeigen, dass das Erlöschen des Deutschen in Krain und des Slowenischen in Kärnten sehr ähnlich erfolgt ist. Auf Grund der deutschen und slowenischen Sprachstruktur ergeben sich durchaus vergleichbare und gleichwertige Übergangsstadien, die man oft als „Mischsprachen“ bezeichnet hat, so in Kärnten als „Windisch“. Deren Sprecher hat man oft als „schwebendes Volkstum“ bezeichnet, doch dieses Wort hat allerdings auch andere (v. a. politische) Nebenbedeutungen. Doch realistisch gesehen handelt es sich hier um keine „Sprachen“, sondern um Sprachformen, die an Einzelpersonen gebunden sind, die sich im Status assimilationis befinden und im Grunde genommen ,zweisprachig“ im wahr(st)en Sinn des Wortes sind, nicht aber um Gruppensprachen oder gar „Dialekte“ im engeren Sinn des Wortes. Somit ist der hier vorgestellte Dialekt ein gutes Beispiel für Sprachkontakt ganz allgemein, der letzten Endes zum Sprachwechsel führt.

Schlüsselwörter: Sprachkontakt, Sprachwechsel, Sprachinseln, Sorica/Zarz, Kärnten, Krain

\section{Abstract \\ SLOVENE-GERMAN LANGUAGE CONTACT IN THE LANGUAGE ENCLAVE SORICA/ZARZ IN COMPARISON TO CARINTHIA}

The Slovene-German language contact in Carniola and the German-Slovene language contact in Carinthia are quite similar. In both countries, there has been a lot of language shift (replacement), such as in Zarz before 1941/45. The language shift in the north and the south of the Karawanks was back-to-front: first the German vocabulary and then the German grammar prevailed in the north, in the south first the Slovene grammar and later the Slovene vocabulary.

The examples given show that the process of extinction of German in Carniola and Slovene in parts of Carinthia was very similar. Due to the German and Slovene language structure, there are comparable and equivalent transition stages, which have often been referred to as "mixed languages", such as "Windisch" in Carinthia. Their speakers have often been characterized as having a rather "floating national identity", but this characterization also has other (especially political) secondary meanings. However, realistically speaking, the so-called "mixed languages" are not "languages" proper, but rather linguistic varieties that are bound to individuals who are in the process of being assimilated and are "bilingual" in the truest sense of the word; they do not refer to group languages or "dialects" in a narrow sense of the word. The situation presented here is therefore a good illustration of language-contact in general, which ultimately leads to language change.

Keywords: language contact, language shift, language enclaves, Sorica/Zarz, Carinthia, Carniola 
Povzetek:

SLOVENSKO-NEMŠKI JEZIKOVNI STIK JEZIKOVNEGA OTOKA SORICA (NEM. ZARZ) V PRIMERJAVI S KOROŠKO

Slovensko-nemški jezikovni stik na Kranjskem in nemško-slovenski na Koroškem sta si v marsičem podobna. $V$ obeh deželah je prihajalo na več mestih do zamenjave jezikov, tako npr. v Sorici že pred letom 1941 oz. 1945. Potek jezikovne menjave severno in južno od Karavank je potekal zrcalno obrnjeno. Na severu se je najprej uveljavilo nemško besedišče in potem še nemška slovnica, na jugu pa se je uveljavila najprej slovenska slovnica in šele kasneje slovensko besedišče.

V članku navedeni primeri kažejo, da je upadanje nemščine na Kranjskem in slovenščine na Koroškem potekalo na podoben način. Na osnovi nemške in slovenske jezikovne strukture so se izoblikovale primerljive in enakovredne prehodne stopnje, ki jih pogosto imenujemo „mešani jeziki“, kot je denimo na Koroškem „vindiški“. Njegove govorce so pogosto označevali kot „,neopredeljeno ljudstvo“, a ta izraz ima tudi druge (predvsem politične) konotacije. Dejansko pa pri tem ne gre za ,jezike“, temveč za jezikovne oblike, vezane na posamezne govorce, za katere velja status assimilationis in ki so pravzaprav „dvojezični“"v pravem pomenu besede, ne pa za jezik določene skupine ali celo „dialekte“ v ožjem pomenu besede. Govor, predstavljen v prispevku, pa je dober primer za jezikovni stik na splošno, ki v končni fazi privede do zamenjave jezika.

Ključne besede: jezikovni stik, menjava jezikov, jezikovni otoki, Sorica/Zarz, Koroška, Kranjska 